\title{
Autoimmune Hepatitis and Primary Biliary Cholangitis Overlap in a Patient with Systemic Lupus Erythematosus and Rheumatoid Arthritis Overlap (Rhupus) - An Unusual Association
}

\author{
Anu Yarky', Priyesh Sharma', Vipan Kumar ${ }^{2}$, Nidhi Chauhan', \\ Priyanka Perasamy ${ }^{1}$ \\ ${ }^{1}$ Department of Medicine, Dr. Rajendra Prasad Government Medical College, Tanda, Kangra, Himachal Pradesh \\ ${ }^{2}$ Department of Orthopaedics, Dr. Rajendra Prasad Government Medical College, Tanda, Kangra, \\ Himachal Pradesh \\ Corresponding Author: Anu Yarky
}

DOI: https://doi.org/10.52403/ijhsr.20220113

\begin{abstract}
The diagnosis of overlap syndrome involving systemic lupus erythematosus (SLE) and autoimmune hepatitis (AIH) isn't easily established due to its similar clinical presentations and biochemical features to those of lupus hepatitis. The term overlap syndrome is typically utilized in the context of overlap of autoimmune hepatitis with PSC (primary sclerosing cholangitis) or PBC (primary biliary cholangitis). Few rare cases of AIH complicated by SLE are reported within the literature. Overlapping of SLE and AIH should be suspected when patients with SLE have abnormal liver function tests or AIH patients present with a rash. Liver biopsy plays a really important role in establishing the medical diagnosis of SLE with liver impairment or overlap with AIH. The prompt diagnosis and adequate treatment plan can improve the disease outcome.
\end{abstract}

Keywords: autoimmune hepatitis, primary biliary cholangitis, systemic lupus erythematosus, overlap syndrome

\section{INTRODUCTION}

Systemic lupus erythematosus (SLE) is a systemic autoimmune disease characterized by multiple clinical criteria and immunological criteria. SLE affects the central nervous system, cardiovascular system, lungs, liver, kidneys, joints, and skin. Autoimmune hepatitis (AIH) presents generally as unresolved inflammation of the liver of unknown etiology and it is characterized by the presence of autoantibodies, hypergammaglobulinemia, and interface hepatitis on histological examination. The diagnosis of $\mathrm{AIH}$ is predicated on characteristic clinical and laboratory findings, abnormal serum globulin levels, the presence of one or more characteristic autoantibodies, and histologic abnormalities.

AIH-SLE overlap syndrome has not been clearly distinguished from lupus hepatitis due to similarities in their clinical and biochemical features, and very few cases of AIH complicated with SLE have been reported. Here we report a case of a patient who presented to our hospital with elevated liver enzymes, jaundice, and arthralgias and was later found to have $\mathrm{AIH}$ and underlying SLE with RA, with no viral or drug-related etiology.

\section{CASE REPORT}

A 22-year-old female was admitted with complaints of fever, yellowish 
discoloration of eyes, dry cough, shortness of breath, and joint pains for 15-20 days. Fever was low grade, not associated with chills, no rigors, no diurnal variation. The patient noticed yellowish discoloration of eyes and dark-colored urine for 15 to 20 days. There was no pruritis, no hematemesis, no Malena, no distension of abdomen, no pain abdomen, no vomiting. The patient also complained of a dry cough associated with shortness of breath for 15 days. The breathlessness decreased on lying down and increased on sitting up. The patient complained of multiple joint pains, bilaterally symmetrical, including bilateral elbow, wrist, and knee joints and small joints of hands, associated with morning stiffness.

History of recurrent joint pains in the past associated with tender swollen joints in the past and recurrent admissions for 6 months for fever and joint pains. History of alopecia and history of oral ulcers for 3 months. History of amenorrhoea for 3 months. History of significant involuntary weight loss of $15 \mathrm{kgs}$ in the past 3 months. Her weight dropped from 45 to $30 \mathrm{kgs}$. No history of loss of appetite. No history of photosensitivity, no h/o rash, no h/o seizures. No history of anti-tubercular drugs intake, no history of intake of steroids. The patient was started on tablet sulfasalazine 45 days ago by another practitioner for arthralgia? rheumatoid arthritis. She took it for 1 week and stopped as she developed gastroenteritis which lasted for 2 weeks. She complained of generalized weakness, easy fatiguability, and arthralgias.

On general examination, she was conscious, cooperative, well oriented to time place, and person. Her vitals were pulse $=88 / \mathrm{min}$, regular, $\mathrm{BP}=104 / 64 \mathrm{~mm}$ of $\mathrm{Hg}, \mathrm{SpO} 2=89 \%$ on room air, respiratory rate $=20 /$ minute. She was pale, icteric, there was no cyanosis, no clubbing, no lymphadenopathy, JVP was not raised, no pedal edema. Her BMI was $11.7 \mathrm{~kg} / \mathrm{m} 2$ (Underweight). There was temporal hollowing, loss of buccal pad of fat, thinning of limbs, dry skin. There was no deformity of small or large joints. Her systemic examination was normal.

Reports: Complete hemogram was suggestive of pancytopenia, $\mathrm{Hb}=6.7$, TLC=3500, PLT=90,000, MCV 99, DLC= $\mathrm{N}_{86} \mathrm{~L}_{9} \mathrm{E}_{2} \mathrm{M}_{3}$, RBS was 80, HBA1C was $4.9 \%$, ESR was 80 , CRP $>12$, serum ferritin $>2000$, LFT suggestive of Conjugated hyperbilirubinemia, Total Bil=7.2, conjugated bil=3.99, AST=306, ALT $=68$, ALP $=1220$, RFT were normal, serum calcium was 7.6, Vitamin D was $10.6 \mathrm{ng} / \mathrm{ml}$, $\mathrm{TtG}$ Ig $\mathrm{A}=7.3 \mathrm{U} / \mathrm{ml}$, urine routine, and cultures were normal. $\mathrm{LH}=1.02 \mathrm{mIU} / \mathrm{ml}$, $\mathrm{FSH}=0.28 \mathrm{mIU} / \mathrm{ml}$, Serum Ceruloplasmin level was normal $0.38 \mathrm{~g} / \mathrm{L}$. Slit-lamp examination was normal, no KF ring was seen.

ANA profile: ANA by Hep2=POSITIVE, end point titre 1:320, anti SMA Negative, AMA POSITIVE, Anti -LKM1 negative, Anti ds DNA positive, C3 $=60 \mathrm{mg} / \mathrm{dl}$, C4=40.6 mg/dl, serum Fibrinogen $=205$ $\mathrm{mg} / \mathrm{dl}, \mathrm{CD} 16$ and CD 25 =low, EBV IgM $=4.14 \mathrm{U} / \mathrm{ml}$ (negative), HBsAg, HBeAg, Anti $\mathrm{HBe}$, Anti $\mathrm{HBc}$ all were negative, HBV DNA positive, CMV IgM 0.47 (negative)

CECT abdomen suggestive of mild to moderate hepatosplenomegaly, no caseous abdominal lymphadenopathy

Chest X-ray was suggestive of bilateral homogenous opacities. (Figure 1)

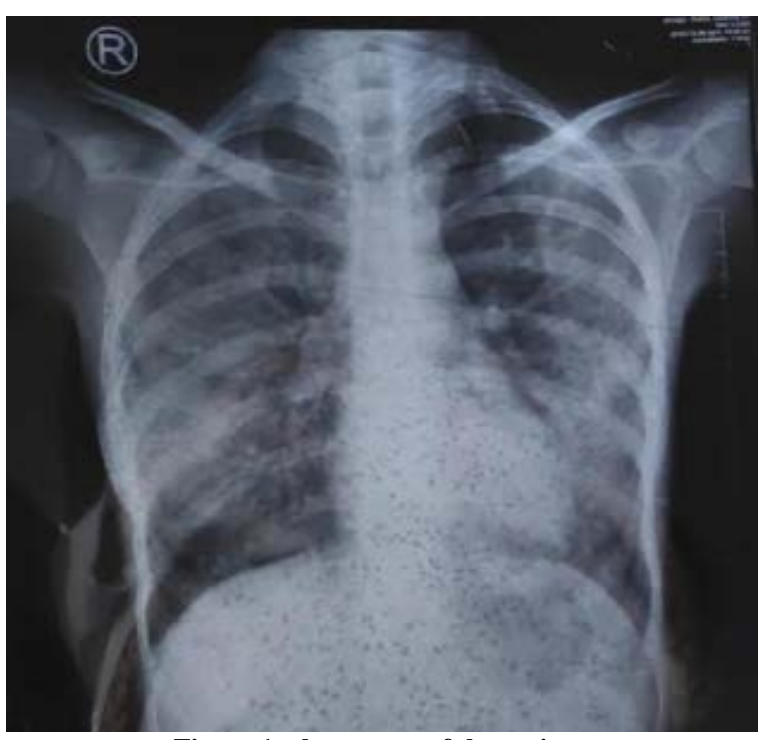

Figure 1: chest $x$-ray of the patient 
CECT thorax was suggestive of bilateral ground-glass opacities and nodular changes on either side with bilateral pleural effusion with adjacent segmental collapse.

MRCP: focal areas of stricturous narrowing in common hepatic duct with focal pruning and dilatation in the right hepatic duct.

Serum iPTH $6.7 \mathrm{pg} / \mathrm{ml}$ (low), serum calcium $6.8 \mathrm{mg} / \mathrm{dl}(\mathrm{low})$, total IGA=7.19 g/dl (raised)Total $\mathrm{IGG}=16.9 \mathrm{~g} / \mathrm{dl}$ (normal), Total IGM=1.12g/dl (normal),

Sputum AFB negative, Mantoux negative, rheumatoid factor negative, anti ccp strongly positive, IgM scrub negative.

The patient underwent a liver biopsy to confirm the diagnosis of Autoimmune hepatitis. Liver histology was suggestive of interface hepatitis, predominantly lymphoplasmacytic infiltrate and resetting of liver cells. There were no bile duct changes of primary biliary cirrhosis or primary sclerosing cholangitis.

\section{RESULT}

Using the SLICC criteria for SLE 2012, the patient was positive for SLE. The positive criteria were positive ANA and Anti dsDNA, presence of oral ulcers, nonscarring alopecia, synovitis, evidence of serositis in the form of bilateral pleural effusion on CT scan chest, leukopenia, and thrombocytopenia.

Using the ACR/EULAR criteria for Rheumatoid arthritis, a diagnosis of Definite RA 6 points was made. The positive criteria were involvement of 2 to 10 large joints, high positive anti-ccp, abnormal CRP and abnormal ESR, and duration of symptoms more than 6 weeks.

Primary Hypoparathyroidism was diagnosed as the patient had low serum PTH level with a concomitant low serum calcium level.

According to the Revised Original AIH Score, the patient scored 10 points i.e., diagnosis Pre-Treatment: Probable AIH.

Positive features/criteria were, Female sex, serum globulins raised 1.5 times upper limit of normal, ANA POSITIVE $>1: 80$, AMA positive, Negative results for all hepatitis viral markers, However, there was a history of recent use of hepatotoxic drugs like sulfasalazine was present, average alcohol intake was $\mathrm{Nil} /<25$ g/day, liver histology was typical of autoimmune hepatitis, other autoimmune diseases like $\mathrm{SLE}+\mathrm{RA}=$ ?RHUPUS was present in the patient and the patient responded completely to the treatment when started on tab azathioprine and prednisolone.

The patient was also evaluated for the presence of Polyglandular autoimmune syndromes but she did not fit into any of the 3 types of PGAs.

PBC may develop in patients who initially present with AIH. Patients with AIH-PBC overlap generally fall into 2 categories. Our patient fell into the first category which is AMA positive without histological evidence of PBC. This variant is sometimes also known as AMA-positive autoimmune hepatitis. The patient was started on tab prednisone $30 \mathrm{mg}$ od and tab hydroxychloroquine $200 \mathrm{mg}$ twice a day. She responded well to the treatment and has been on regular follow up for 6 months, without relapse. Her steroids have been tapered to the lowest possible dose now and we plan on doing a follow-up liver biopsy one year later to assess histological improvement along with the biochemical response.

\section{DISCUSSION}

Autoimmune hepatitis is a disease of the liver parenchyma that can present in acute or chronic forms. The disease mostly arises due to environmental triggers in genetically susceptible individuals. Clinical and biochemical remission is achievable in up to $85 \%$ of cases. Treatment options also include alternative immunosuppression strategies and Liver transplantation in patients with acute liver failure or complications of end-stage liver disease, including hepatocellular carcinoma. Variant or overlapping syndromes should be considered when unexpected disease features arise.[1] 
Diagnosis of AIH is difficult due to the absence of specific diagnostic markers and therefore the large heterogeneity of its clinical, laboratory, and histological features. Clinical manifestations can vary from asymptomatic to severe or rarely fulminant hepatitis. Hypergammaglobulinemia with a selective elevation of IgG is found in most cases. Histology (interface hepatitis, emperipolesis, and hepatic rosette formation) and autoantibodies detection although not pathognomonic, are still the hallmark of a timely diagnosis. AIH remains a major diagnostic challenge and it should be considered in every case in the absence of viral, metabolic, genetic, and toxic etiology of chronic or acute hepatitis. We as physicians got to become more conversant in various disease expressions and therefore in the interpretation of liver histology and autoimmune serology to derive maximum benefit for the patient.[2]

Autoimmune hepatitis was formerly known as Lupoid hepatitis because it had some clinical and serological similarities to SLE. Autoimmune liver disease is comparatively uncommon in patients with SLE. E.g., during a retrospective study of 377 adult patients with SLE, only five (1.3 percent) had histologically confirmed liver damage with autoimmune features [3]. Another retrospective analysis of 147 SLE patients found that 4.7 percent of patients were diagnosed with autoimmune hepatitis [4].

Patients with autoimmune hepatitis may present with a coexisting extrahepatic disorder, which can even be autoimmunemediated. For example, associated common autoimmune disorders include autoimmune thyroiditis, rheumatoid arthritis, type 1 diabetes mellitus, ulcerative colitis, celiac disease, and systemic lupus erythematosus. [5]

Liver dysfunction occurs in approximately $50 \%$ of patients with systemic lupus erythematosus (SLE), and patients with SLE and elevated liver enzymes can present a difficult medical diagnosis. Lupus hepatitis and autoimmune hepatitis are 2 immunologic conditions involving the liver, which may have a similar clinical, laboratory, and systemic presentations, resulting in a difficult diagnosis. Physicians need to be aware of these 2 hepatic diseases as diagnosis and appropriate therapy needs to start early in the disease course to prevent progression to advanced liver disease. [6]

Diagnostic criteria - The diagnosis of autoimmune hepatitis is often made during a patient with a compatible clinical presentation when the subsequent features are present:

- A minimum of one elevated serum aminotransferase, typically (but not always) an aspartate aminotransferase (AST) and/or alanine aminotransferase (ALT) level a minimum of twice the upper limit of the reference range.

- A minimum of one positive laboratory test: increased total IgG or gammaglobulin levels, and/or serologic markers (antinuclear antibodies [ANA], antismooth muscle antibodies [ASMA] at a titre of at least 1:40, anti-liver/kidney microsomal-1 [anti-LKM-1] antibodies, anti-liver cytosol antibody-1 [ALC-1], or anti-soluble liver/liver pancreas [antiSLA/LP] antibodies).

- Exclusion of diseases that have an identical presentation, particularly viral hepatitis, drug-induced liver injury, and alcoholic liver disease.

When a liver biopsy is obtained, the diagnosis can be confirmed by histology showing interface hepatitis and/or a predominantly lymphoplasmacytic infiltrate.

Disease classification - Based on the autoantibody profiles, patients can be categorized into two disease subtypes: type 1 or type 2 AIH.

\section{Type 1 autoimmune hepatitis -}

Autoantibodies characteristic of type 1 autoimmune hepatitis are:

- Antinuclear antibody (ANA).

- Anti-smooth muscle antibody (ASMA; approximately 65 percent of patients). 
- Anti-actin antibodies.

- Anti-mitochondrial antibodies (AMA; rarely positive in patients without primary biliary cholangitis overlap).

- Anti-soluble liver antigen/liver pancreas antibody-antigen

(SLA/LP; approximately 10 to 30 percent of adults).

- Anti-single-stranded and anti-doublestranded DNA (25 to 35 percent of patients)

- Atypical perinuclear anti-neutrophil cytoplasmic antibodies.

Type 2 autoimmune hepatitis Autoantibodies characteristic of type 2 autoimmune hepatitis are antibodies to LKM-1 alone or accompanied by anti-liver cytosol antibody-1 (ALC-1). Positive titers are defined as >1:20 for ANA and antismooth muscle antibody (ASMA), whereas titers of 1:10 may be considered positive for anti-LKM-1

Autoantibody negative autoimmune hepatitis - Approximately 20 percent of patients who present with all the features of autoimmune hepatitis lack circulating ANA, ASMA, or anti-LKM-1 antibodies. These patients are usually considered having autoantibody negative autoimmune hepatitis or cryptogenic chronic hepatitis.

Diagnostic scoring systems - A scoring system developed to standardize the diagnosis for population-based studies and clinical trials has had limited value in individual patients. A less complicated system using simplified criteria for individual patients is predicated upon titers of autoantibodies, IgG levels, liver histology, and therefore the exclusion of viral hepatitis.

A probable diagnosis of autoimmune hepatitis is made if the total points are 6, while a definite diagnosis is made if the total points are $\geq 7$. A potential limitation of the scoring system is the relative lack of standardization of some of the autoantibody tests.[7]

TREATMENT: Our general approach is to begin treatment with glucocorticoid monotherapy. For patients with mild disease (e.g., asymptomatic patients with aminotransferase levels $<10$ times the upper limit of normal), lower-dose prednisone monotherapy (e.g., $20 \mathrm{mg}$ per day) is an alternative. In patients with moderate to severe disease who are at increased risk for side effects from glucocorticoids (e.g., elderly, those with brittle diabetes, osteoporosis, emotional lability, a history of psychosis, or poorly controlled hypertension) a combination of lowerdose prednisone (30 mg per day) and azathioprine (50 $\mathrm{mg}$ per day) is preferred for initial treatment.

The American Association for the Study of Liver Diseases (AASLD) recommends initial therapy with either glucocorticoid monotherapy or a combination of a glucocorticoid and azathioprine, whereas the British Society of Gastroenterology (BSG) and European Association for the Study of the Liver (EASL) recommend initial treatment with a glucocorticoid and azathioprine rather than glucocorticoid monotherapy

Subsequent management depends on the patient's response to the initial treatment (remission, incomplete response, failed treatment, drug intolerance) and whether the patient relapses if treatment is withdrawn. In some cases, treatment can be withdrawn completely whereas other patients require maintenance therapy. For patients who do not respond to standard treatment, secondline treatment with other immunosuppressants has proven to be effective. Treatment should be aimed at biochemical remission of the disease i.e., normalization of transaminases and immunoglobulin $G$ and histological improvement on one to two-year followups. Patients should be monitored during the first months of treatment intensively to monitor side effects, assess symptoms, and individualize treatment. [8]

Many experimental therapies are being explored in the management of AIH. Drugs that block B cell-activating factors of the tumor necrosis factor family (BAFF) and tumor necrosis factor $\alpha$ are currently in 
Anu Yarky et.al. Autoimmune hepatitis and primary biliary cholangitis overlap in a patient with systemic lupus erythematosus and rheumatoid arthritis overlap (rhupus) -an unusual association.

clinical trials for the treatment of AIH. There is also evidence for targeting inflammatory pathways to control other autoimmune conditions, such as blockade of IL1 and IL6 and Janus-associated kinase (JAK) inhibitors. The therapeutic landscape of AIH will likely change over the coming years and new treatment approaches offering lower corticosteroid use and aiming to restore immune self-tolerance will come up.[9]

\section{CONCLUSION}

Liver test abnormalities have been described in up to $60 \%$ of patients with systemic lupus erythematosus (SLE) at some point during their disease. Prior treatment with potentially hepatotoxic drugs or viral hepatitis is commonly considered to be the main cause of liver disease in SLE patients. However, in rare cases elevated liver enzymes may be due to concurrent autoimmune hepatitis (AIH). To distinguish whether the patient has primary liver disease with associated autoimmune clinical and laboratory features resembling SLE - such as AIH - or the elevation of liver enzymes is a manifestation of SLE remains a difficult challenge for the treating physician. [10] Physicians need to be aware of the various clinical criteria of autoimmune hepatitis and also look for the presence of extrahepatic autoimmune conditions associated with it. Timely diagnosis and early treatment can greatly improve the prognosis of such patients.

Acknowledgements: We would like to thank the patient and the medicine department for their cooperation and assistance.

\section{Declarations}

Funding: No funding sources.

Conflict of Interest: None declared.

Ethical Approval: Not required.

\section{REFERENCES}

1. Heneghan MA, Yeoman AD, Verma S, Smith AD, Longhi MS. Autoimmune hepatitis. Lancet. 2013 Oct 26;382(9902):1433-44. doi: 10.1016/S01406736(12)62163-1. Epub 2013 Jun 14. PMID: 23768844.

2. Gatselis NK, Zachou K, Koukoulis GK, Dalekos GN. Autoimmune hepatitis, one disease with many faces: etiopathogenetic, clinico-laboratory and histological characteristics. World J Gastroenterol. 2015 Jan 7;21(1):60-83. doi: 10.3748/wjg.v21.i1.60. PMID： 25574080; PMCID: PMC4284362.

3. Irving KS, Sen D, Tahir H, Pilkington C, Isenberg DA. A comparison of autoimmune liver disease in juvenile and adult populations with systemic lupus erythematosus-a retrospective review of cases. Rheumatology (Oxford). 2007 Jul;46(7):1171-3. doi: 10.1093/rheumatology/kem108. Epub 2007 May 8. PMID: 17488749.

4. Efe C, Purnak T, Ozaslan E, Ozbalkan Z, Karaaslan Y, Altiparmak E, Muratori P, Wahlin S. Autoimmune liver disease in patients with systemic lupus erythematosus: a retrospective analysis of 147 cases. Scand J Gastroenterol. 2011 Jun;46(6):732-7. doi: 10.3109/00365521.2011.558114. Epub 2011 Feb 25. PMID: 21348808.

5. Bittencourt PL, Farias AQ, Porta G, Cançado EL, Miura I, Pugliese R, Kalil J, Goldberg AC, Carrilho FJ. Frequency of concurrent autoimmune disorders in patients with autoimmune hepatitis: effect of age, gender, and genetic background. J Clin Gastroenterol. 2008 Mar;42(3):300-5. doi: 10.1097/MCG.0b013e31802dbdfc. PMID: 18223493.

6. Adiga A, Nugent K. Lupus Hepatitis and Autoimmune Hepatitis (Lupoid Hepatitis). Am J Med Sci. 2017 Apr;353(4):329-335. doi: 10.1016/j.amjms.2016.10.014. Epub 2016 Nov 4. PMID: 28317620.

7. Terziroli Beretta-Piccoli B, Mieli-Vergani G, Vergani D. Serology in autoimmune hepatitis: A clinical-practice approach. Eur J Intern Med. 2018 Feb;48:35-43. doi: 10.1016/j.ejim.2017.10.006. Epub 2017 Oct 19. PMID: 29056396.

8. Pape S, Schramm C, Gevers TJ. Clinical management of autoimmune hepatitis. United European Gastroenterol J. 2019 Nov;7(9):1156-1163. doi: 10.1177/2050640619872408. Epub 2019 
Anu Yarky et.al. Autoimmune hepatitis and primary biliary cholangitis overlap in a patient with systemic lupus erythematosus and rheumatoid arthritis overlap (rhupus) -an unusual association.

Aug 25. PMID: 31700628; PMCID: PMC6826525.

9. Halliday N, Dyson JK, Thorburn D, Lohse AW, Heneghan MA. Review article: experimental therapies in autoimmune hepatitis. Aliment Pharmacol Ther. 2020 Oct;52(7):1134-1149.

doi: 10.1111/apt.16035. Epub 2020 Aug 14. PMID: 32794592.

10. Beisel C, Weiler-Normann C, Teufel A, Lohse AW. Association of autoimmune hepatitis and systemic lupus erythematodes: a case series and review of the literature.

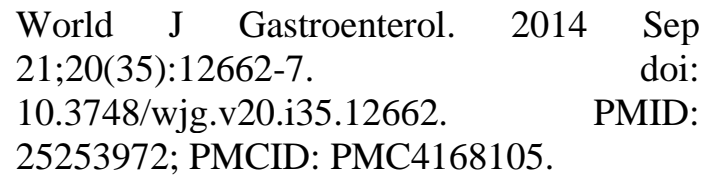

World J Gastroenterol. 2014 Sep 21;20(35):12662-7. doi: 10.3748/wjg.v20.i35.12662. PMID: 25253972 ; PMCID: PMC4168105.

How to cite this article: Yarky A, Sharma P, Kumar $\mathrm{V}$ et.al. Autoimmune hepatitis and primary biliary cholangitis overlap in a patient with systemic lupus erythematosus and rheumatoid arthritis overlap (rhupus) -an unusual association. Int J Health Sci Res. 2022; 12(1): 92-98. DOI: https://doi.org/10.52403/ ijhsr.20220113 